УДК 538.9:536.6

\title{
ВЛИЯНИЕ МЕТОДОВ ПОЛУЧЕНИЯ ПОЛИМЕРНЫХ МИКРО- И НАНОКОМПОЗИТОВ НА ИХ ТЕПЛОФИЗИЧЕСКИЕ СВОЙСТВА
}

\author{
Долинский А.А. ${ }^{1}$, академик НАН Украины, Фиалко Н.М. ${ }^{1}$, член-корреспондент НАН Украины, \\ Динжос P.B. ${ }^{2}$, канд. физ.-мат. наук, Навродская Р.А., канд. техн. наук \\ ${ }^{1}$ Институт технической теплофизики НАН Украинь,, ул. Желябова, 2 а, Киев, 03680, Украина \\ ${ }^{2}$ Николаевский национальный университет им. В.А.Сухомлинского, ул. Никольская, 24, Николаев, \\ 540030, Украина
}

Наведено результати досліджень теплофізичних властивостей високотеплопровідних полімерних композитів на основі поліпропілену, наповнених вуглецевими нанотрубками або частками алюмінію, при використанні для одержання даних композитів методів, що базуються на змішуванні компонентів в сухому вигляді і в розплаві полімеру. При цьому представлено матеріали щодо закономірностей впливу зазначених методів на теплопровідні властивості розглянутих композитів, їх теплоємність, щільність, пористість, ступінь кристалічності полімерної матриці та iн. Дано пояснення механізмів такого впливу на основі аналізу ефективності утворення перколяційних структур.

Библ. 8, рис. 2 .

Ключевые слова: полимерные микро- и нанокомпозиты, углеродные нанотрубки, теплофизические свойства, методы получения композитов.

$c_{p}$ - удельная массовая теплоемкость, Дж/(кг·К); $\Delta^{p} H_{m}, \Delta H_{m c}-$ энтальпия плавления композита и полностью кристаллического полимера, Дж/кг;

$T$ - температура, К;

$\lambda$ - коэффициент теплопроводности, Вт/(м·К);

П, $\Pi_{p m}-$ пористость композитов и полимерной

Введение

Bce более широкое использование полимерных микро- и нанокомпозитов связано, как известно, с их улучшенными свойствами по сравнению с ненаполненными полимерами. Потребности дальнейшего развития данного направления обусловливают необходимость углубленного изучения влияния различных
The results of studies of thermophysical properties of the highly conducting polymer composites based on polypropylene filled with carbon nanotubes or particles of aluminum, at using methods for acquisition of these composites based on mixing the components in dry form and in the polymer melt are given. This contains materials about the patterns of influence of these methods on the heat-conducting properties of the composites, their heat capacity, density, porosity, degree of crystallinity of the polymer matrix, and so forth are offered. The explanations of the mechanisms of this influence based on analysis of the effectiveness of formation of the percolation structures are presented. $\rho_{p}, \rho_{f}-$ плотность полимерной матрицы и наполнителя, кг $/ \mathrm{M}^{3}$;

$\chi$ - степень кристалличности композита, \%;

$\omega-$ массовая доля наполнителя, \%;

УНТ - углеродные нанотрубки.

факторов на основные характеристики таких композитов. Согласно результатам ряда исследований эти характеристики существенно зависят от методов получения композиционных материалов [1 - 7]. Данное обстоятельство определяет актуальность сравнительного анализа этих методов в плане установления закономерностей их влияния на структуру и свойства композитов. Особый матрицы, \%; 
интерес представляет проведение соответствующих исследований для высокотеплопроводных композитов, перспективы применения которых связаны с изготовлением деталей теплоэнергетического оборудования, установок химической, пищевой, перерабатывающей промышленности и пр.

\section{Постановка задачи и методика проведения исследований}

Настоящая статья посвящена, главным образом, экспериментально-теоретическим исследованиям закономерностей влияния методов получения высокотеплопроводных полимерных микро- и нанокомпозитов на их теплофизические характеристики.

При проведении экспериментов использовались два наиболее широко распространенных в инженерной практике метода получения полимерных композитов:

- метод А, основанный на смешении находящихся в сухом виде компонентов с применением магнитной мешалки и ультразвукового диспергатора при дальнейшем горячем прессовании полученной композиции;

- метод В, базирующийся на смешении компонентов в расплаве полимера с применением экструдера при дальнейшем придании композиту необходимой формы способом горячего прессования.

Что касается второго из указанных методов, то в рамках данного исследования использовалась одна из его модификаций, в которой применяются так называемые дисковые экструдеры, характеризующиеся рядом известных достоинств в сравнении с традиционными шнековыми аппаратами.

Рассматриваемые методы применялись для получения композитов на основе полипропилена, наполненных УНТ или частицами алюминия. При проведении исследований массовая доля наполнителей изменялась от 0,3 до 10 \%. Описание методов изготовления указанных наполнителей и их характеристик дано в [8]. Здесь же приведены методики, используемые для определения теплофизических свойств получаемых композитов.

\section{Результаты исследований и их анализ}

Остановимся вначале на рассмотрении результатов экспериментальных исследований зависимости коэффициентов теплопроводности полимерных композитов от массовой доли наполнителей (УНТ или микрочастиц алюминия) при использовании для получения данных композитов методов, основанных на смешении компонентов в сухом виде и в расплаве полимеpa. Согласно приведенным на рис. 1 данным применение второго из указанных методов позволяет получать полимерные композиты с существенно более высокими, чем в случае первого метода, теплопроводящими свойствами.

Обращает на себя внимание также тот факт, что увеличение значений $\lambda$, связанное с использованием метода $B$, оказывается более существенным для полимерного композита, наполненного УНТ, по сравнению с микрокомпозитом, наполненным алюминием. Так, отличия значений $\lambda$ для двух рассматриваемых методов при массовой доле наполнителей $5 \%$ составляют $28,4 \mathrm{BT} /(\mathrm{M} \cdot \mathrm{K})$ при использовании в качестве наполнителя УНТ, а при наполнении полимера микрочастицами алюминия - лишь 14,7 Вт/(м·К).

Как видно из рис. 1, расхождения коэффициентов теплопроводности полимерных композитов, полученных различными методами, существенно зависят от массовой доли наполнителя Ф. Для обоих рассматриваемых композитов эти отклонения являются незначительными в диапазоне изменения $\omega$ от 0,3 до $2 \%$. С увеличением $\omega$ они возрастают, достигая максимального значения при массовой доле наполнителей, равной $4 \%$. При этом коэффициенты теплопроводности композитов, полученных методом $B$, превышают соответствующие величины для композитов, полученных на основе метода $A$, в случае наполнения полимера УНТ и алюминием в 4,0 и 4,2 раза соответственно. Далее с повышением массовой доли наполнителей от 4 до 10 \% наблюдается тенденция к уменьшению расхождений в значениях $\lambda$, отвечающих разным методам получения композитов. Причем данная тенденция оказывается существенно более ярко выраженной для микрокомпозитов, наполненных алюминием. Здесь указанные расхождения уменьшаются от 17,6 Вт/(м·K) при $\omega=4 \%$ до $4,1 \mathrm{BT} /(\mathrm{м} \cdot \mathrm{K})$ при $\omega=10 \%$. Для композитов, наполненных УНТ, это уменьшение являет- 
ся не столь значительным - от $31,2 \mathrm{BT} /(\mathrm{м} \cdot \mathrm{K})$ до 26,2 Bт/(м·K).

Рассмотрим вкратце особенности влияния методов получения композитов на эффекты резкого изменения величины их коэффициентов теплопроводности при определенных значениях доли наполнителей $\omega$. Как видно из рис. $1, \sigma$, на кривых $\lambda=f(\omega)$ для обоих рассматриваемых композитов наблюдаются два скачка коэффициента теплопроводности. Первый из них отвечает образованию из частиц наполнителей перколяционных кластеров, представляющих собой своеобразные теплопроводящие каналы, второй - формированию перколяционной сетки, являющейся высокотеплопроводной средой. Что касается положения указанных скачков на кривых $\lambda=f(\omega)$, то оно, как следует из приведенных данных, изменяется в зависимости от метода получения композита. При этом отвечающие данным скачкам значения $\omega$ - так называемые перколяционные пороги, для метода $B$ смещаются в область меньших концентраций наполнителя. Так, для обоих рассматриваемых композитов первый перколяционный порог равняется 1,15 и 0,55 \% соответственно при получении данных композитов методами $A$ и $B$. Второй перколяционный порог отвечает концентрации УНТ, равной $4,2 \%$, и частиц алюминия - 4,6 \% для метода $A$. Данный порог снижается при использовании метода $B$ до 3,15 и до 2,85 \% при наполнении полимера УНТ и микрочастицами алюминия.

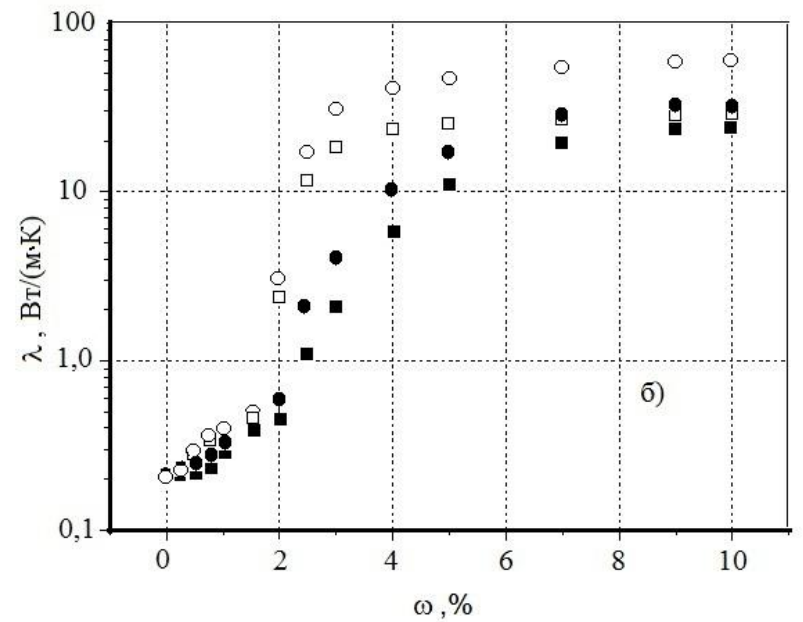

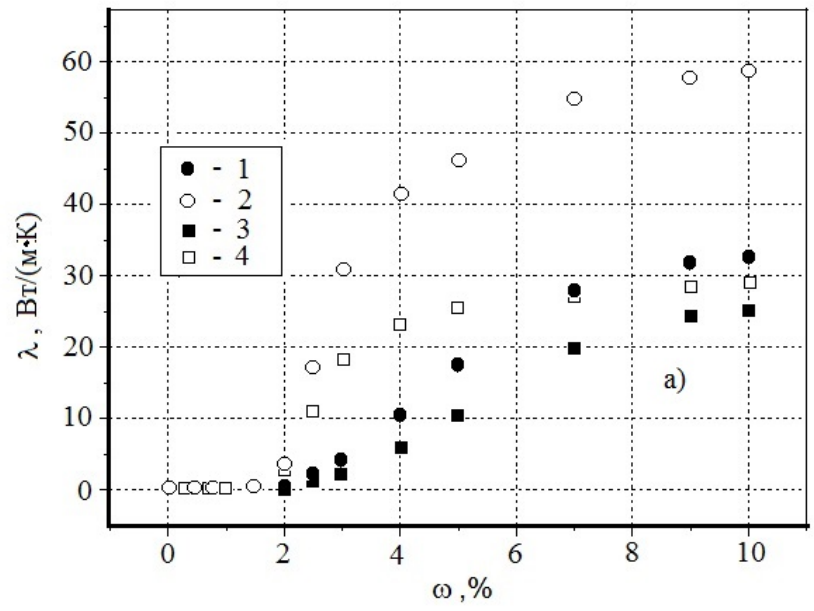

Рис. 1. Зависимость коэффициента теплопроводности полимерных композитов на основе полипропилена, полученных с использованием методов смешения компонентов в сухом виде $(1,3)$ и в расплаве полимера $(2,4)$, при наполнении полимерной матрицы УНТ (1, 2) и микрочастицами алюминия (3, 4): а), б) - линейная и логарифмическая шкала по оси ординат соответственно.

Описанная картина влияния методов получения полимерных композитов на их теплопроводящие свойства связана со следующими обстоятельствами. Как показали результаты выполненных исследований, применение для получения композитов метода $B$ обеспечивает более равномерное распределение наполнителя в полимерной матрице. Это в свою очередь обуславливает большую эффективность образования из частичек наполнителя непрерывных перко- ляционных кластеров и перколяционных сеток, ответственных за повышение коэффициента теплопроводности материалов.

Относительно установленного факта большей чувствительности композита, наполненного УНТ, к методу его получения, то он объясняется более существенным влиянием степени равномерности распределения наполнителя в объеме полимера на образование указанных перколяционных структур. Действительно, ввиду того, что 
длина углеродной нанотрубки значительно превышает ее диаметр, и к тому же силы электромагнитного взаимодействия между трубками существенно меньше, чем между микрочастицами алюминия, образование перколяционных структур из УНТ при повышении равномерности их распределения в полимерной матрице происходит более эффективно в сравнении с микрочастицами алюминия.

Перейдем далее к рассмотрению результатов экспериментальных исследований по определению удельной теплоемкости полимерных микро- и нанокомпозитов, получаемых различными методами. Как видно из табл. 1, 2, значения $c_{p}$, отвечающие разным методам, заметно отличаются лишь в области плавления полимера. При этом величины $c_{p}$ для композитов, полученных методом $B$, являются в указанной области более низкими для всех значений ные данные свидетельствуют также о том, что влияние метода получения композита на значения его удельной теплоемкости существеннее проявляются при наполнении полимера УНТ.

Таблица 1. Температурная зависимость удельной теплоемкости $c_{p}(\kappa Д ж /(\kappa г \cdot К))$ полимерного композита на основе полипропилена, наполненного УНТ, при его получении с использованием метода смешения компонентов в сухом виде (метод $A$ ) и в расплаве полимера (метод $B$ ) для различных значений массовой доли наполнителя

\begin{tabular}{|c|c|c|c|c|c|c|c|c|c|c|c|}
\hline \multirow{2}{*}{$\begin{array}{c}\text { Метод } \\
\text { получения } \\
\text { композита }\end{array}$} & \multirow{2}{*}{$\begin{array}{c}\text { Содержание } \\
\text { наполнителя, } \\
\%\end{array}$} & \multicolumn{10}{|c|}{$\mathrm{T}, \mathrm{K}$} \\
\hline & & 300 & 360 & 420 & 425 & 430 & 435 & 440 & 442 & 445 & 450 \\
\hline \multirow{3}{*}{$A$} & 0,3 & 1,77 & 2,30 & 3,62 & 4,17 & 4,97 & 5,93 & 6,84 & 6,93 & 6,23 & 3,16 \\
\hline & 3 & 1,89 & 2,38 & 3,60 & 4,11 & 4,85 & 5,73 & 6,58 & 6,66 & 6,01 & 3,18 \\
\hline & 10 & 1,92 & 2,40 & 3,60 & 4,11 & 4,82 & 5,68 & 6,51 & 6,59 & 6,00 & 3,17 \\
\hline \multirow{3}{*}{$B$} & 0,3 & 1,75 & 2,26 & 3,56 & 4,09 & 4,88 & 5,81 & 6,71 & 6,80 & 6,16 & 3,11 \\
\hline & 3 & 1,88 & 2,28 & 3,29 & 3,71 & 4,33 & 5,05 & 5,75 & 5,82 & 5,32 & 2,93 \\
\hline & 10 & 1,91 & 2,28 & 3,23 & 3,63 & 4,21 & 4,90 & 5,56 & 5,62 & 5,16 & 2,90 \\
\hline
\end{tabular}

На основе результатов исследований температурной зависимости теплоемкости $c_{p}$ выполнена оценка степени кристалличности полимера $\chi$ в композиционных материалах, полученных различными методами. Величина $\chi$ определялась с использованием этих данных по соотношению [9]. $\chi=\frac{\Delta H_{m}}{\Delta H_{m c}} \cdot 100 \%$.

Результаты расчетов степени кристалличности полипропилена представлены на рис. 2. Как следует из приведенных данных, для рассматриваемых полимерных композитов степень кристалличности полипропилена уменьшается с ростом массовой доли наполнителей. Причем это уменьшение является более значительным для композитов, полученных методом $B$. Что же касается степени кристалличности полипропилена, наполненного УНТ и микрочастицами алюми- ния, то во всем исследуемом диапазоне изменения $\omega$ она оказывается меньшей при наполнении полипропилена УНТ для обоих рассматриваемых методов получения композитов.

Установленные закономерности влияния метода получения композитов на степень кристалличности полимерной матрицы непосредственно связаны с уровнем равномерности распределения наполнителя, который реализуется при использовании того или иного метода. Как уже отмечалось, более высокий уровень данной равномерности имеет место при использовании метода $B$. В этих условиях наблюдается большая эффективность образования перколяционных кластеров и сеток, которые являются стерическими препятствиями для формирования в полимерной матрице кристаллических структур. Иными словами меньший уровень кристаллизации полимерной матрицы для композитов, полученных 
методом $B$, объясняется образованием из частиц наполнителя более разветвленных перколяционных систем ввиду большей равномерности распределения данных частиц в объеме матрицы. Именно отмеченная большая разветвленность и усложняет процесс кристаллизации полимера.

На рис. 3 представлены результаты экспе- риментальных исследований по определению температурной зависимости плотности рассматриваемых полимерных композитов, полученных различными методами, при фиксированной массовой доле наполнителя $\omega=3 \%$. Как видно, для обоих исследуемых методов во всем диапазоне изменения температур плотность

Таблица 2. Температурная зависимость удельной теплоемкости $c_{p}$ (кДж/(кг·К)) полимерного композита на основе полипропилена, наполненного микрочастицами алюминия, при его получении с использованием метода смешения компонентов в сухом виде (метод $A$ ) и в расплаве полимера (метод $B$ ) для различных значений массовой доли наполнителя

\begin{tabular}{|c|c|c|c|c|c|c|c|c|c|c|c|}
\hline \multirow{2}{*}{$\begin{array}{c}\text { Метод } \\
\text { получения } \\
\text { композита }\end{array}$} & \multirow{2}{*}{$\begin{array}{c}\text { Содержание } \\
\text { наполнителя, } \\
\%\end{array}$} & \multicolumn{10}{|c|}{$\mathrm{T}, \mathrm{K}$} \\
\hline & & 300 & 360 & 420 & 425 & 430 & 435 & 440 & 442 & 445 & 450 \\
\hline \multirow{3}{*}{$A$} & 0,3 & 1,76 & 2,29 & 3,62 & 4,18 & 4,98 & 5,94 & 6,87 & 6,96 & 6,30 & 3,15 \\
\hline & 3 & 1,78 & 2,31 & 3,64 & 4,19 & 4,99 & 5,94 & 6,86 & 6,94 & 6,29 & 3,17 \\
\hline & 10 & 1,80 & 2,32 & 3,63 & 4,17 & 4,97 & 5,91 & 6,82 & 6,91 & 6,27 & 3,17 \\
\hline \multirow{3}{*}{$B$} & 0,3 & 1,76 & 2,29 & 3,61 & 4,16 & 4,97 & 5,93 & 6,85 & 6,99 & 6,28 & 3,14 \\
\hline & 3 & 1,90 & 2,37 & 3,55 & 4,03 & 4,75 & 5,59 & 6,41 & 6,49 & 5,91 & 3,13 \\
\hline & 10 & 1,96 & 2,41 & 3,53 & 3,99 & 4,67 & 5,48 & 6,26 & 6,33 & 5,78 & 3,13 \\
\hline
\end{tabular}

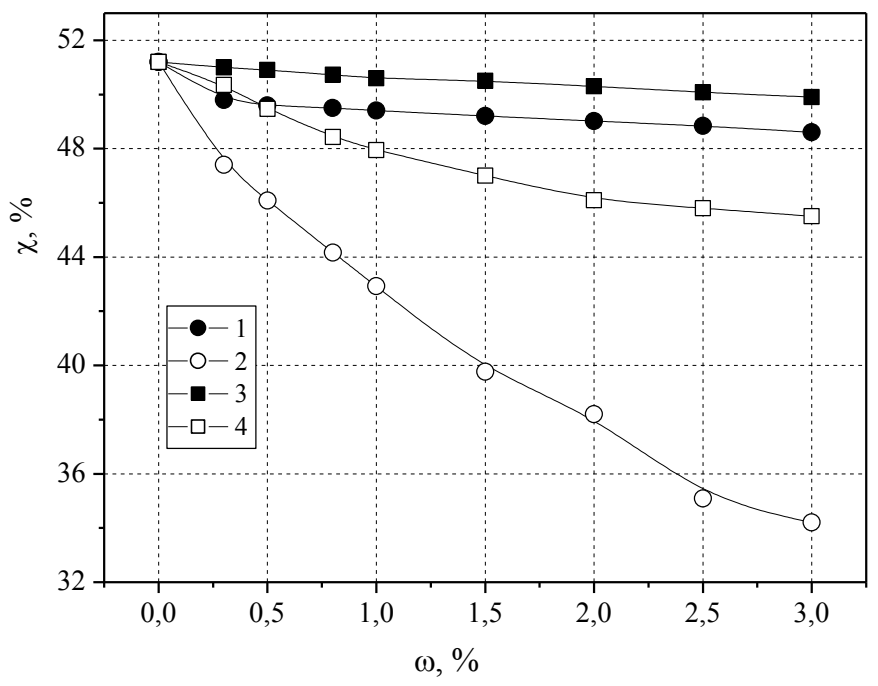

Рис. 2. Зависимость степени кристалличности полипропилена от массовой доли наполнителя в композитах, полученных с использованием метода смешения компонентов в сухом виде $(1,3)$ и в расплаве полимера (2, 4), при наполнении полипропилена УНТ $(1,2)$ и микрочастицами алюминия $(3,4)$.

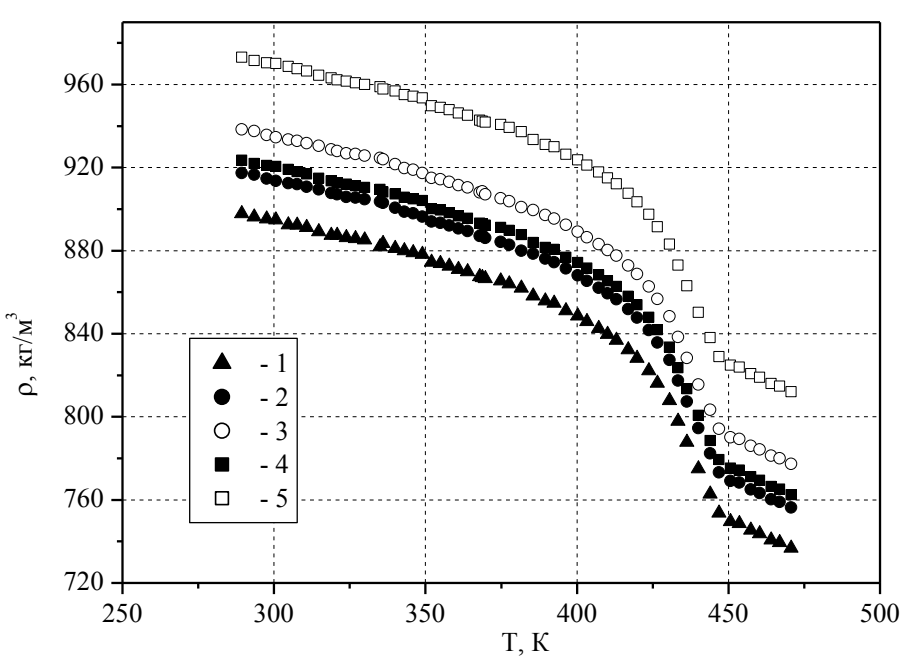

Рис. 3. Температурная зависимость плотности полипропилена (1) и композиционных материалов на его основе, полученных с использованием метода смешения компонентов в сухом виде $(2,4)$ и в расплаве полимера

$(3,5)$, при наполнении полипропилена УНТ $(4,5)$ и частицами алюминия $(2,3)$ для $\omega=3 \%$. 
композитов, наполненных УНТ, оказывается выше соответствующих значений плотности при наполнении полимера частицами алюминия. При этом, однако, соотношение плотностей рассматриваемых наполнителей является противоположным, а именно, плотность УНТ составляет 2200 кг/ $\mathrm{M}^{3}$, а алюминия $2700 \kappa г / \mathrm{M}^{3}$. Таким образом, большая плотность композитов с УНТ обусловливается не уровнем плотности наполнителя, а механизмами формирования данных композиционных материалов. Действительно, в рассматриваемых условиях в аморфных зонах полимера ввиду электромагнитного взаимодействия частиц наполнителя с полимерной матрицей наблюдается уплотнение материала в целом. Причем такое уплотнение происходит несколько более интенсивно при наполнении полимера УНТ, поскольку эти силы в данном случае оказываются более значительными из-за особенностей формы трубок и их огром- ной удельной площади поверхности $(190$ м²/г).

Что же касается влияния на плотность композитов методов их получения, то большей плотности отвечает метод $B$. Это связано с описанной выше картиной увеличения уровня равномерности распределения наполнителей в матрице и, как следствие, с большей разветвленностью формирующихся перколяционных структур при реализации данного метода. Наличие таких структур определяет усиление электромагнитного взаимодействия между наполнителями и полимерной матрицей, что и приводит к уплотнению материала.

Важно также отметить, что согласно данным выполненных экспериментальных исследований отличия плотности композитов, полученных разными методами, оказываются более существенными для композитов, наполненных УНТ.

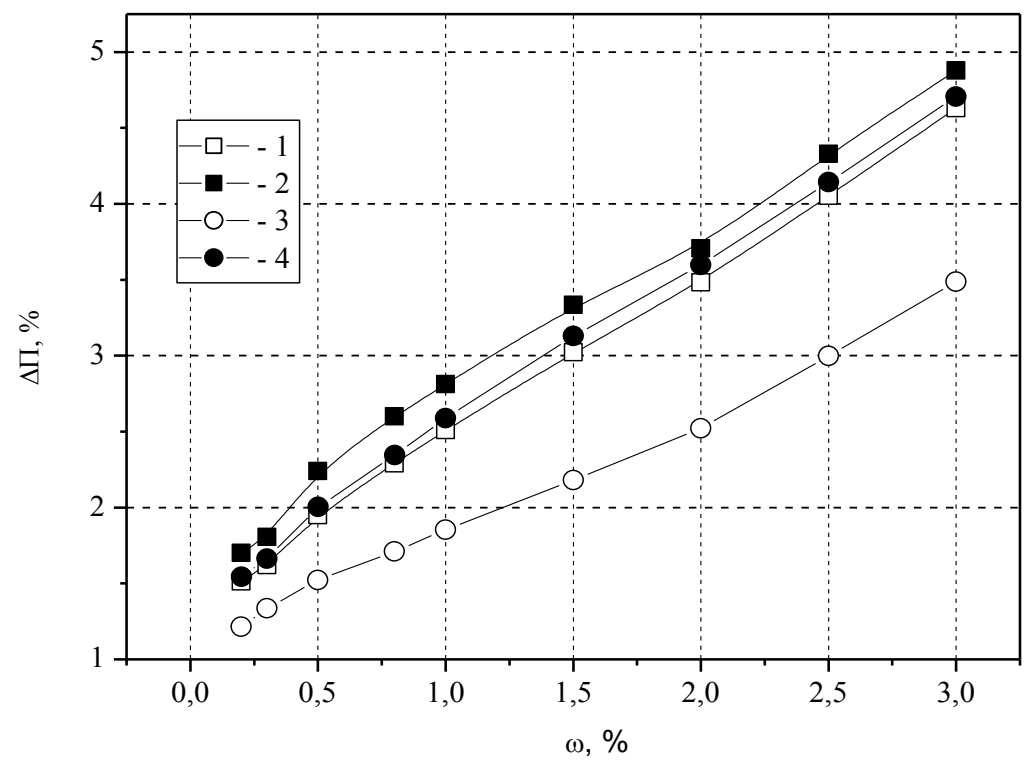

Рис. 4. Зависимость от массовой доли наполнителей величины приращения пористости АП полимерных композитов, полученных с использованием метода смешения компонентов в сухом виде $(2,4)$ и в расплаве полимера $(1,3)$, при наполнении композита УНТ $(3,4)$ и частицами алюминия $(1,2)$.

Рис. 4 иллюстрирует зависимость от массовой доли наполнителя величины приращения пористости композитов $\Delta П$, которое обусловлено введением наполнителя в полимерную матрицу $\left(\Delta \Pi=\Pi-\Pi_{\mathrm{pm}}\right)$. Здесь значения $\Pi$ определялись в соответствии с [10] по формуле

$$
\Pi=1-\frac{\rho-\left[\frac{\rho_{f}}{\rho_{p}}(1-\omega)\right] \rho_{p}}{\left[1-\frac{\rho_{f}}{\rho_{p}(1-\omega)}\right] \rho_{f}} .
$$


Полученные данные свидетельствуют о том, что пористость композитов возрастает с увеличением доли наполнителя. При этом указанное увеличение является несколько более значительным для композитов, наполненных частицами алюминия, и к тому же проявляется в большей мере при реализации первого из рассматриваемых методов получения композиционных материалов - метода смешения компонентов в сухом виде.

\section{Выводы}

1. Выполнены экспериментальные исследования по установлению закономерностей влияния на теплофизические характеристики полимерных микро- и нанокомпозитов методов их получения. Для композитов на основе полипропилена, наполненных УНТ или частицами алюминия, исследования проведены в условиях варьирования массовой доли наполнителей $\omega$ от 0,3 до $10 \%$ при использовании двух методов получения таких композитов - метода $A$, основанного на смешении компонентов в сухом виде, и метода $B$, базирующегося на их смешении в расплаве полимера.

2. Проведен сравнительный анализ рассматриваемых методов получения полимерных композитов в плане их влияния на теплопроводящие свойства материалов. Показано, что при использовании метода $B$ могут быть получены полимерные микро- и нанокомпозиты с существенно более высокими, чем в случае метода $A$, значениями коэффициента теплопроводности $\lambda$. Установлен факт большей чувствительности полимерных композитов, наполненных УНТ, к методу их получения. Обнаружено, что при использовании метода $B$ величины массовой доли наполнителя, отвечающие резкому изменению $\lambda$ - так называемые перколяционные пороги, смещаются в область меньших значений $\omega$.

3. На основе экспериментальных исследований установлены закономерности изменения удельной теплоемкости $c_{p}$ рассматриваемых композитов от температуры при использовании различных методов их получения в широком диапазоне изменения массовой доли наполнителей. В частности, показано, что величины $c_{p}$, отвечающие разным методам получения композитов, заметно отличаются только в области плавления полимерной матрицы. При этом данные отличия оказываются более значительными для композитов, наполненных УНТ, и возрастают с повышением массовой доли наполнителя.

C использованием результатов экспериментальных исследований теплоемкости композитов выполнена оценка степени кристалличности полимерной матрицы. Установлено, что последняя уменьшается с ростом доли наполнителей и это уменьшение является более существенным для материалов, полученных с использованием метода $B$, и к тому же для композитов, наполненных УНТ.

4. Для рассматриваемых полимерных микро- и нанокомпозитов выполнены экспериментальные исследования по определению их плотности в зависимости от методов получения данных композитов, температуры и массовой доли наполнителей. Установлено, что значения плотности композитов, полученных с использованием метода $B$, несколько выше в сравнении с соответствующими величинами для метода $A$. Показано также, что композиты, наполненные УНТ, при прочих равных условиях характеризуются более высокой плотностью, чем при наполнении полипропилена частицами алюминия.

\section{ЛИТЕРАТУРА}

1. Sathyanarayana S. Thermoplastic Nanocomposites with Carbon Nanotubes/ S. Sathyanarayana, C. Hübner. - Structural Nanocomposites. - 2013. - P. 19-60.

2. Huang Y. Y. Dispersion of Carbon Nanotubes: Mixing, Sonication, Stabilization, and Composite Properties / Yan Yan Huang, Eugene M. Terentjev. Polymers. - 2012. - V. 4.- P. 275-295.

3. Peng-Cheng Ma. Dispersion and functionalization of carbon nanotubes for polymerbased nanocomposites: A review / Peng-Cheng Ma, Naveed A. Siddiqui, Gad Marom, Jang-Kyo Kim.Composites: Part A. - 2010. - V. 41.- P. 1345-1367.

4. Дінжос Р. В. Вплив методу введення наповнювача на теплофізичні властивості систем на основі термопластичних полімерів та вуглецевих нанотрубок / Р. В. Дінжос, Е. А. Лисенков, Н. М. Фіалко, В. В. Клепко // Фізична інженерія поверхні. - 2014. - Т.12, №4. - С. 446-453. 
5. Kouichi T. A Molecular dynamics study on thermal conductivity of thin epoxy polymer sandwiched between alumina fillers in heatdissipation composite material / Kouichi Tanaka, Shuji Ogata, Ryo Kobayashi, Tomoyuki Tamura, Takahisa Kouno. - International Journal of Heat and Mass Transfer. - 2015. - V. 89.- P. 714-723.

6. Sathyanarayana $S$. Influence of peroxide addition on the morphology and properties of polypropylene-multiwalled carbon nanotube composites. / S. Sathyanarayana, C. Hübner. Composites: Part A Compos. Sci. Technol. - 2013. - V. 84.- P. 78-85.

7. Lebovka N. I. Phase behavior, microstructure and percolation of poly(ethylene glycol) filled by multiwalled carbon nanotubes and organophilic montmorillonite. / Lebovka N. I., Lysenkov E. A.,
GoncharukA. I., Gomza Yu. P., Klepko V. V., Boiko Yu. P. - Journal of Composite Materials. - 2011. Vol. 45, No. 24. - P. 2555-2566.

8. Долинский А. А. Теплофизические свойства полимерных микро- и нанокомпозитов на основе поликарбоната / А. А. Долинский, Н. М. Фиалко, Р. В. Динжос, Р. А. Навродская // Промышленная теплотехника.- 2015. - №2. C.12-19

9. Беритейн В. А. Дифференциальная сканирующая калориметрия в физикохимии полимеров/ В. А. Берштейн, В. М. Егоров. - Л.: Химия, 1990. $-256 \mathrm{c}$.

10.Колупаев Б.С. Релаксационные и термические свойства наполненных полимерных систем / Под ред. С.Я. Френкеля. - Львов: ЛГУ, 1980. -203 c. 


\section{INFLUENCE OF RECEIPT METHODS OF POLYMERIC MICRO- AND NANOCOMPOSITES ON THEIR THERMOPHYSICAL PROPERTIES}

\section{Dolinskiy A.A.', Fialko N.M. ${ }^{1}$, Dinzhos R.V. ${ }^{2}$, Navrodskaya R.A.}

${ }^{1}$ Institute of Engineering Thermophysics, National Academy of Sciences of Ukraine, 2a, Zhelyabova str., Kyiv, 03680, Ukraine

${ }^{2}$ Nikolaev National University. named after V.A. Sukhomlinskiy, 24, Nikolska str., Mykolaev, 540030, Ukraine

The results of studies of thermophysical properties of the highly conducting polymer composites based on polypropylene filled with carbon nanotubes or particles of aluminum, at using methods for acquisition of these composites based on mixing the components in dry form and in the polymer melt are given. This contains materials about the patterns of influence of these methods on the heat-conducting properties of the composites, their heat capacity, density, porosity, degree of crystallinity of the polymer matrix, and so forth are offered. It is shown that by using the method of mixing the components in the polymer melt compared to the method of mixing in a dry form composites provided a much higher heat-conducting properties, somewhat lower values of specific heat (mostly in region of polymer fusion) the higher density and lower degree of crystallinity of the polymer matrix. The explanations of the mechanisms of this influence based on analysis of the effectiveness of formation of the percolation structures are presented.

References 10, fig. 4. tabl. 2.

Key words: polymer micro- and nanocomposites, carbon nanotubes, thermophysical properties, methods for composites.

\footnotetext{
1. Sathyanarayana $\quad S$ Thermoplastic Nanocomposites with Carbon Nanotubes / S. Sathyanarayana, C. Hübner. - Structural Nanocomposites. - 2013. - P. 19-60.

2. Huang Y. Y. Dispersion of Carbon Nanotubes: Mixing, Sonication, Stabilization, and Composite Properties / Yan Yan Huang, Eugene M. Terentjev. -
}

Polymers. - 2012. - V. 4.- P. 275-295.

3. Peng-Cheng Ma. Dispersion and functionalization of carbon nanotubes for polymerbased nanocomposites: A review / Peng-Cheng Ma, Naveed A. Siddiqui, Gad Marom, Jang-Kyo Kim. Composites: Part A. - 2010. - V. 41.- P. 1345-1367.

4. Dinzhos $R$. $V$. Influence input filler method on thermal properties of systems based on thermoplastic polymers and carbon nanotubes / R. V. Dinzhos, E. A. Lisenkov, N. M. Fialko, V. V. Klepko // Physical Surface Engineering. - 2014. - V.12, №4. - P. 446453. (Ukr)

5. Kouichi T. A Molecular dynamics study on thermal conductivity of thin epoxy polymer sandwiched between alumina fillers in heatdissipation composite material / Kouichi Tanaka, Shuji Ogata, Ryo Kobayashi, Tomoyuki Tamura, Takahisa Kouno. - International Journal of Heat and Mass Transfer. - 2015. - V. 89.- P. 714-723.

6. Sathyanarayana $S$. Influence of peroxide addition on the morphology and properties of polypropylene-multiwalled carbon nanotube composites. / S. Sathyanarayana, C. Hübner. Composites: Part A Compos. Sci. Technol. - 2013. - V. 84.- P. 78-85.

7. Lebovka N. I. Phase behavior, microstructure and percolation of poly (ethylene glycol) filled by multiwalled carbon nanotubes and organophilic montmorillonite. / Lebovka N. I., Lysenkov E. A., GoncharukA. I., Gomza Yu. P., Klepko V. V., Boiko Yu. P. - Journal of Composite Materials. - 2011. Vol. 45, No. 24. - P. 2555-2566.

8. Dolinskiy A. A. Thermal properties of polymer micro- and nanocomposites based on polycarbonate / A, A. Dolinskiy, N. M. Fialko, R. V. Dinzhos, R. A. Navrodskaya // Industrial teplotehnika.-2015. №2. - P. 12-19. (Rus)

9. Bernstein $V$. A. Differential Scanning Calorimetry in physical chemistry of polymers / V. A. Bernstein, V. M. Egorov. - L .: Chemistry, 1990. (Rus)

10. Kolupaev B.S. Relaxation and thermal properties of filled polymer systems / Ed. S.Y. Frenkel. - Lviv: LSU, 1980. - 203 p. (Rus)

Получено 25.05.2015 Received 25.05.2015 